\title{
Research Paper: Investigating the Relationship Between Individual and Clinical Characteristics and Executive Dysfunction of Multiple Sclerosis Individuals
}

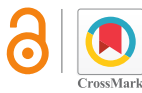

\section{Maryam Pouramiri ${ }^{1}$, Mojtaba Azimian $^{1}$ (1), ${ }^{*}$ Nazila Akbarfahimi $^{1}$ (1), Ebrahim Pishyareh ${ }^{1}$ (1), Samaneh Hossienzadeh ${ }^{2}$ (1)}

1. Department of Occupational Therapy, University of Social Welfare and Rehabilitation Sciences, Tehran, Iran

2. Department of Biostatistics, University of Social Welfare and Rehabilitation Sciences, Tehran, Iran.

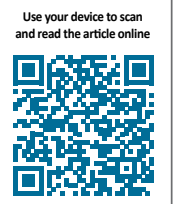

Citation Pouramiri M, Azimian M, Akbarfahimi N, Pishyareh E, Hossienzadeh S. [Investigating the Relationship Between Individual and Clinical Characteristics and Executive Dysfunction of Multiple Sclerosis Individuals (Persian)]. Archives of Rehabilitation. 2019; 20(2):114-123. http://dx.doi.org/10.32598/ri.20.2.114

http://dx.doi.org/10.32598/rj.20.2.114

Received: 10 Dec 2018

Accepted: 25 Mar 2019

Available Online: $01 \mathrm{Jul} 2019$
Keywords:

Multiple Sclerosis,

Executive function, Individual characteristic, Clinical characteristic

\section{ABSTRACT}

Objective Multiple Sclerosis (MS) is the most prevalent neurological progression that often affects young adults. Cognitive impairment is a frequent symptom of the disease. One cognitive domain is an executive function. Executive function is important in individuals' cognitive skills, adaptive behaviors, and life satisfaction. Thus, accurately recognizing and investigating the factors affecting it greatly helps with improving MS. The current study investigated the relationship between individual and clinical characteristics and executive dysfunction in patients with $\mathrm{MS}$

Materials \& Methods This was a cross-sectional, descriptive and analytical study conducted on MS patients referring to Rofeydeh Rehabilitation Hospital in 2017. In total, 71 patients were selected through unpredictable sampling method. MS patients and their families provided written consent forms for participation in the study, and the subjects' data were kept confidential. Moreover, the patients were allowed to discontinue study participation as desired. The inclusion criteria were a diagnosis of MS disease based on McDonald's criteria by a neurologist, the lack of memory impairments, including Alzheimer's disease, non-congenital syndromes like Down syndrome, no thyroid dysfunction, the lack of consuming drugs that affect memory and cognitive function like benzodiazepines, and the lack of severe vision and motor impairments. Exclusion criteria included the lack of patient's cooperation with completing the questionnaire and performing the test, dissatisfaction with continuing the collaboration, and the emergence of an acute problem in the patient that enables him/her to continue cooperation. In this research, instruments such as Pittsburgh Sleep Quality Index (PSQI), Fatigue Assessment Scale (FAS), Beck's Depression Inventory (BDI), Expanded Disability Status Scale (EDSS), and Delis-Kaplan Executive Function System (D-KEFS) test were used. The collected data were analyzed in SPSS. For data analysis, general linear regression was used.

Results The obtained results suggested that age, disease duration, and fatigue had a significant reverse relationship with executive functions (descriptive and categorical) $(P>0.05)$. Executive function (classification) had a significant relationship with the first symptom of the onset of disease ( $P>0.05)$

Conclusion We investigated the relationship between individual and clinical characteristics and the executive function in MS patients. A significant relationship was observed between the first sign of the disease onset and the level of performance. People who initially demonstrated vision problems had a weaker performance than others. This can be considered as an important finding in the early stages of diagnosis and the development of therapeutic programs to postpone cognitive complications.

\section{* Corresponding Author: \\ Nazila Akbarfahimi, PhD.}

Address: Occupational Therapy, University of Social Welfare and Rehabilitation, Tehran, Iran.

Tel: +98 (21) 22180037

E-Mail: fahimi1970@yahoo.com 


\section{بررسى رابطه ويثَّىهاى فردى و بالينى با اختلالات عملكرد اجرايى افراد مالتيبل اسكلروزيس}

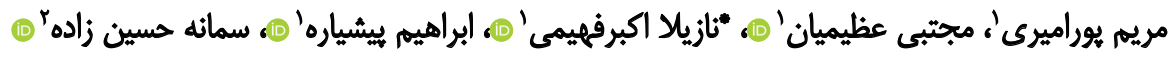

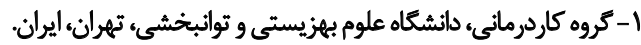

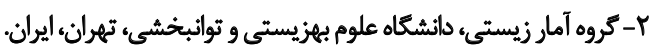

\section{حكSי}

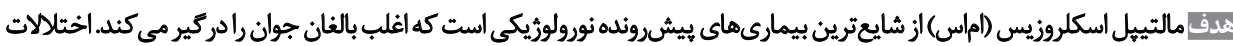

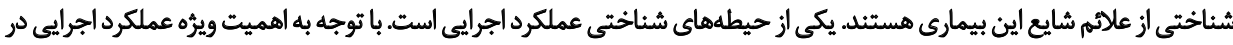

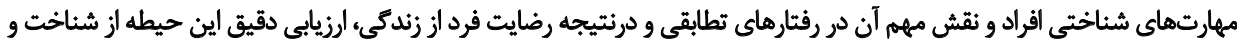

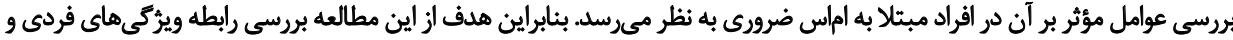

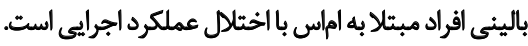

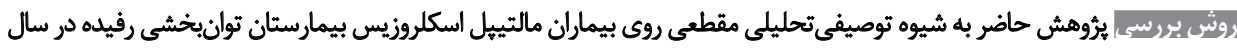

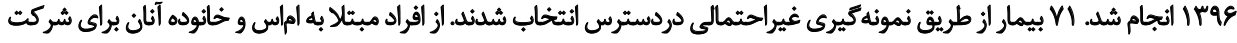

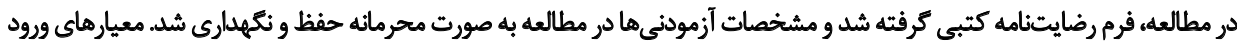

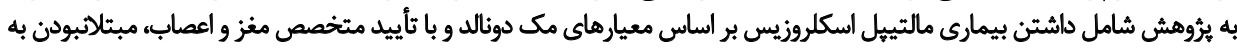

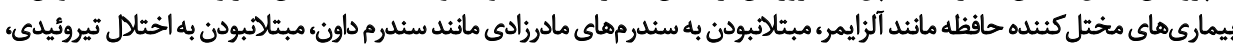

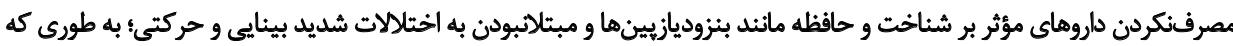

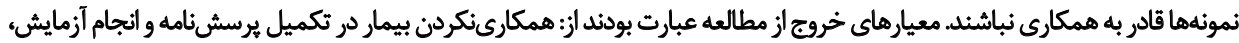

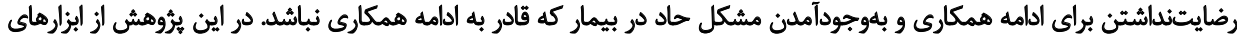

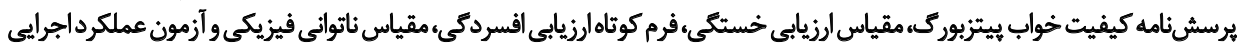

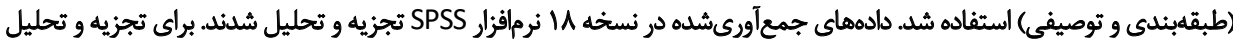

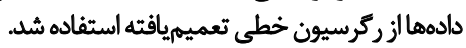

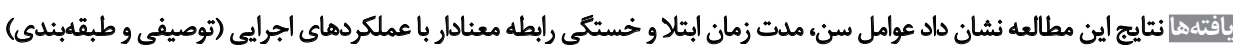

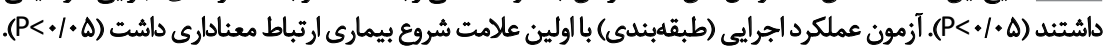

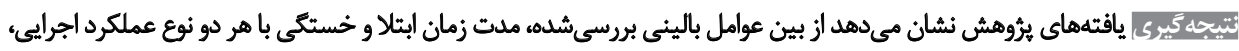

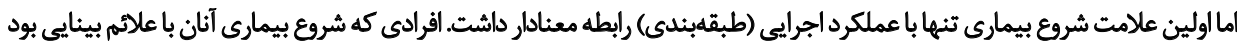

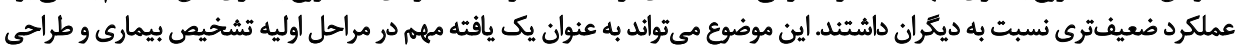

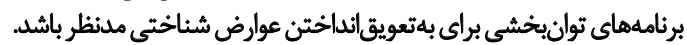

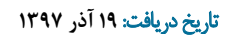

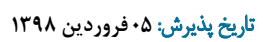

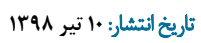

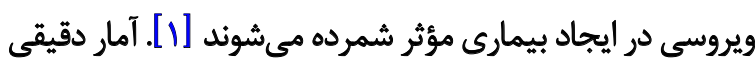

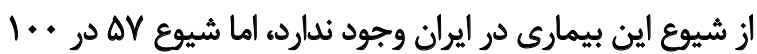

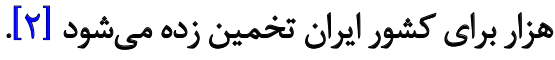

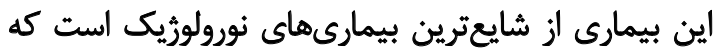

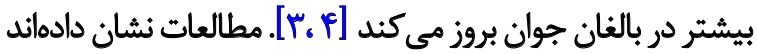

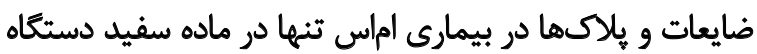
عصبى مركزى نيستند، بلكه در كورتكس و ماده خاديا خاكسترى 다.

مقdad

مالتييل اسكلروزيس' (ام|س) يك بيماري بيشرونده دستكاه

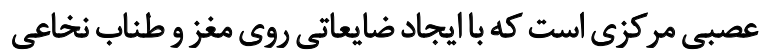

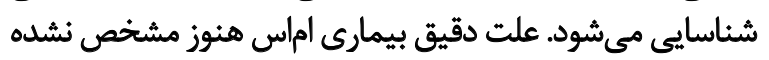

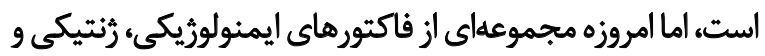

1. Multiple Sclerosis

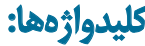

مالتييل اسكلروزيس، اليدوران

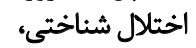

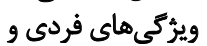

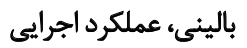


اين مطالعه بررسى رابطه اختلال عملكرد اجرايى باويرُكى هاى فردى و بالينى افراد مبتلا به اماس بودي إنتال

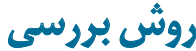

يُروهش حاضر به شيوه توصيفى تحليلى مقطعى روى بيماران

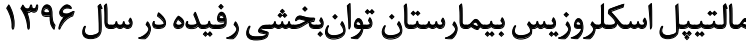

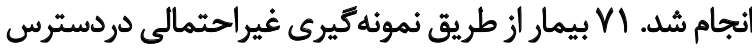

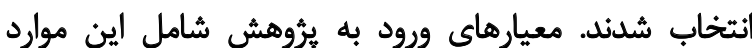

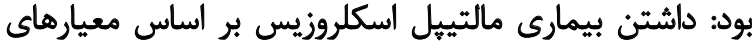

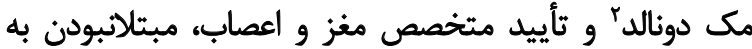

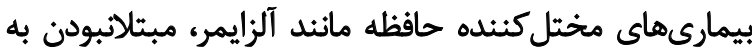

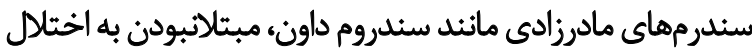

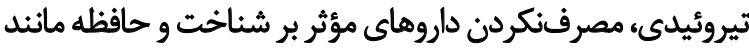

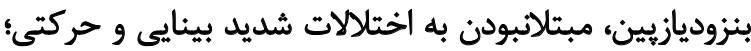

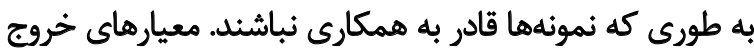

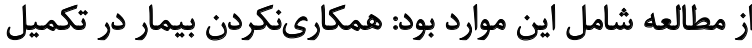

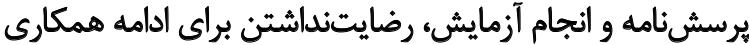
و بلوجودآمدن مشكل حاد دآنمايث، رضيمار (ناتوانى در ادامه همكارىى).

بيماران بر اساس ثرونده و طبق معيارهاى ورود به مطالعه

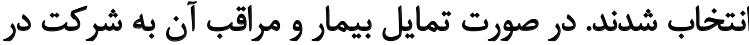

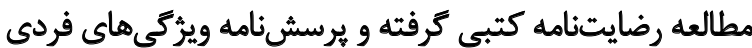

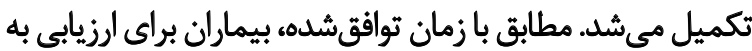

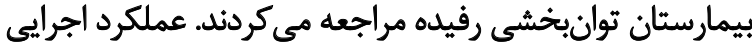

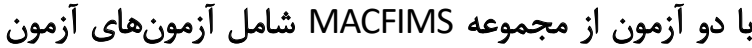

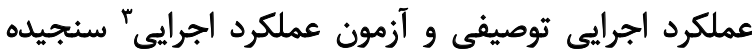

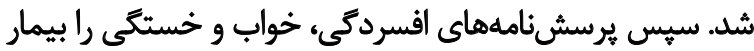

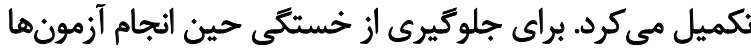

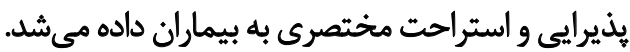

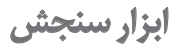

\section{يرسش بامه اطلاعات زمينهاى}

اين برسشنامه محققساخته شامل مشخصات سن، جنسيت،

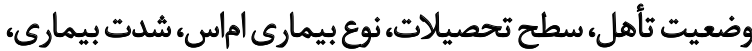

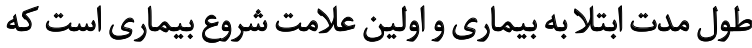

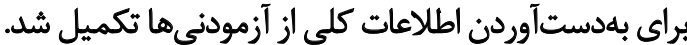

\section{يرسش يرامه ارزيابى اختلالات خواب؟}

اين يرسشنامه نكرش افراد را بيرامون كيفيت خواب در جهار

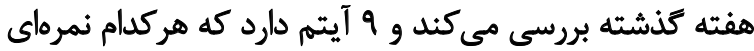

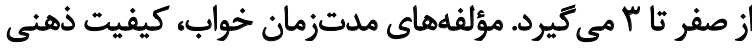

\section{McDonald}

3. D-KEFS sorting \& D-KEFS descriptive

4. Pittsburgh Sleep Quality Index (PSQI)

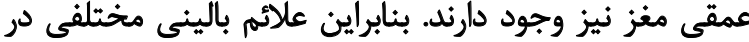

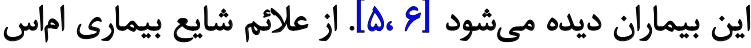

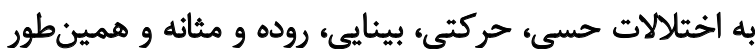
اختلالات شناختى و هيجانى مى توان اشاره كرد [f] اختلالات شناختى از جمله اختلالات شايع در اين بيمارى

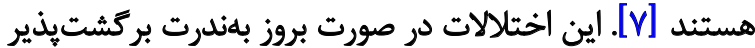

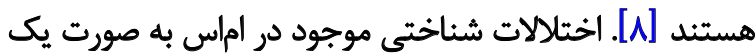

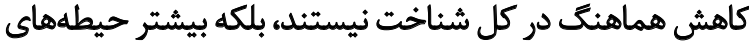

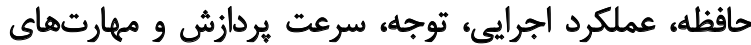

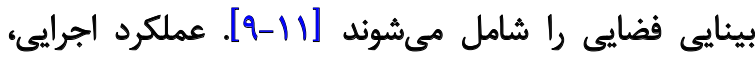

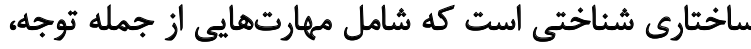

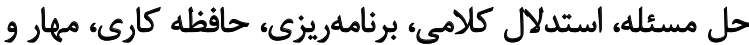

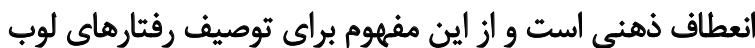
فرونتال و يره فرونتال استفاده مي أشود [ب]

تحقيقات نشان داده است اختلالات شناختى در بيماران مبتلا

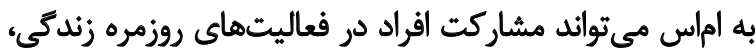

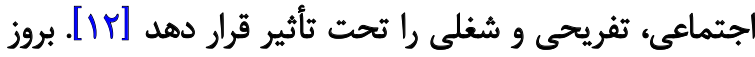

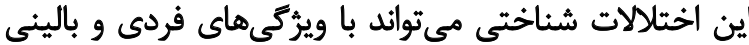

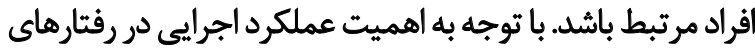

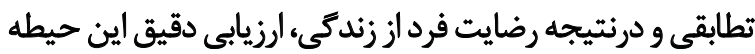

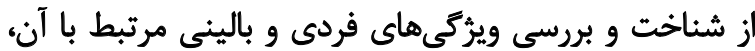

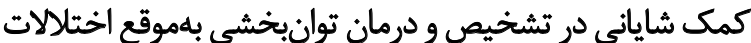

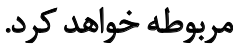

در اكثر مطالعات كذُشته در اين زمينهه ارتباط شناخت بابرخي

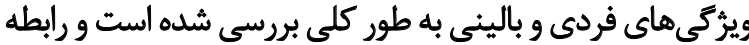

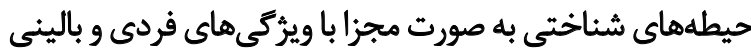

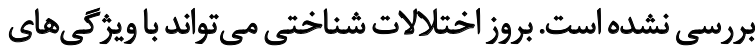

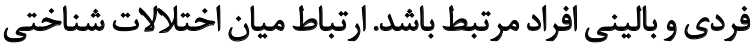

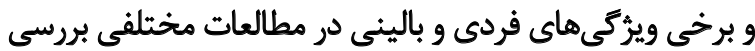

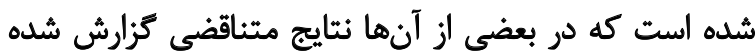

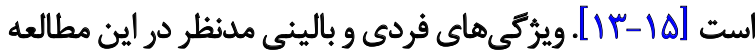

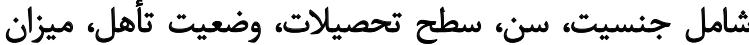

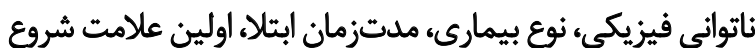
بيمارى، خستكّى، اختلالات خواب و افسردكّى هستند.

بررسى ارتباط بروز اختلال عملكرد اجرايى با ويرّكىهاى

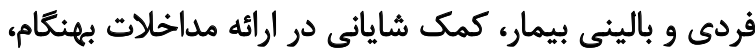

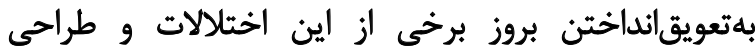

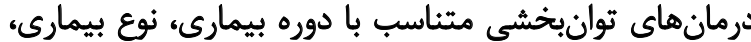

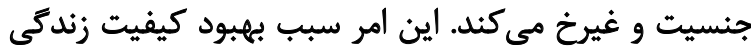

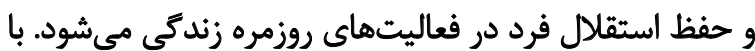

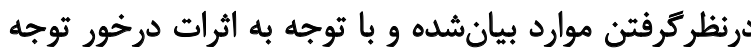

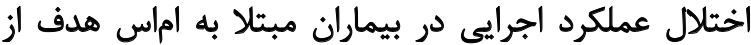


متغيرهاى مستقل (ويرُكىهاى فردى و بالينى) بر متغيرهاى

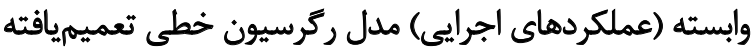

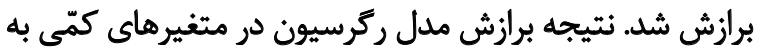

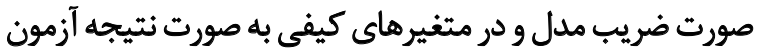

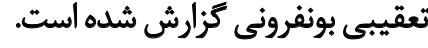

يافتهاهن

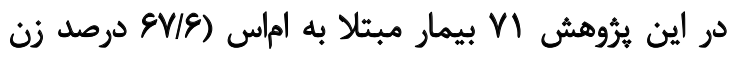

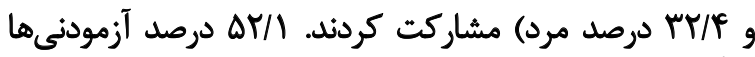

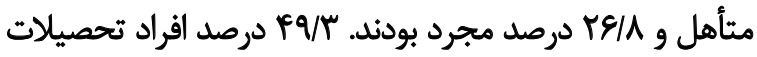

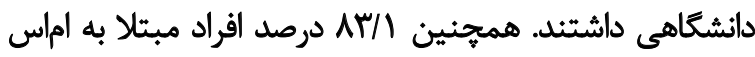

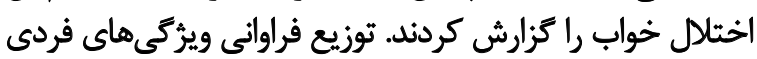

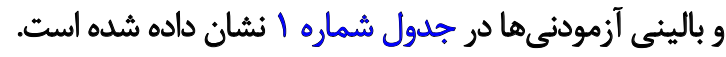

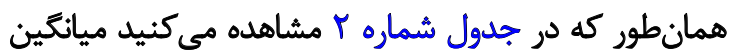

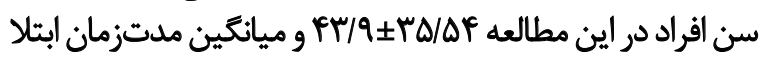

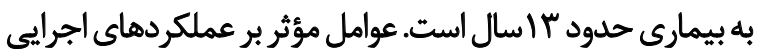

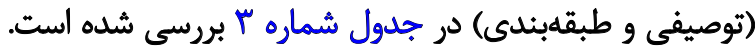

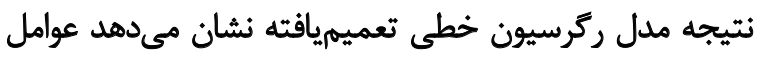

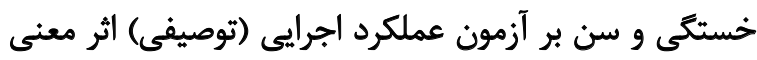

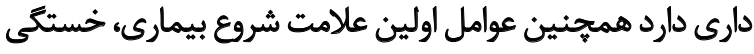

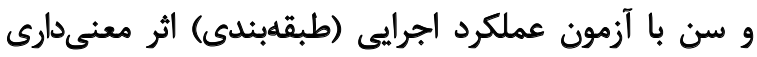

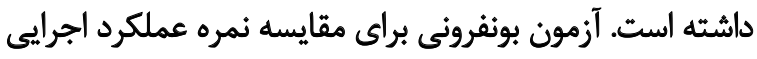

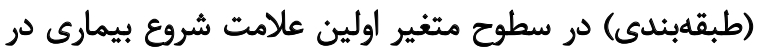
جدول شماره f آمده است.

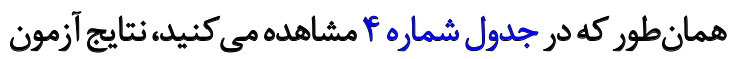

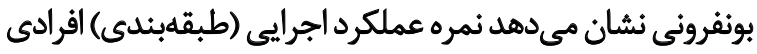

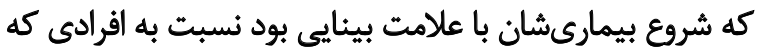

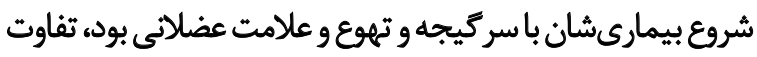

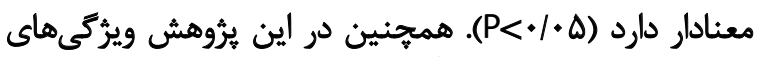

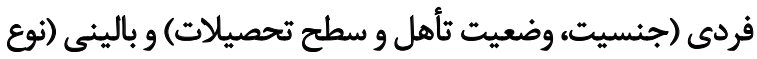

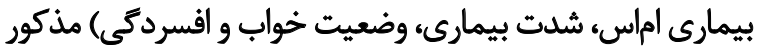

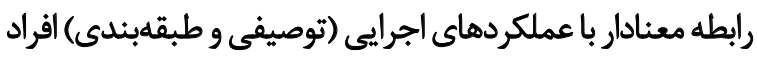

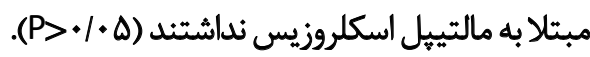

بحث

هدف اين مطالعه بررسى رابطه اختلال عملكردهاى اجرايى

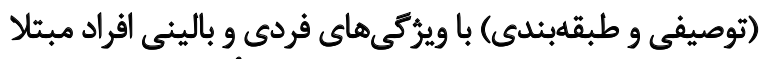

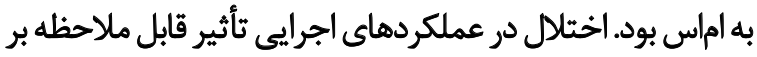

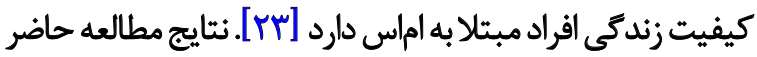

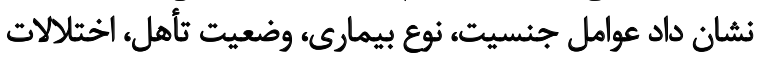

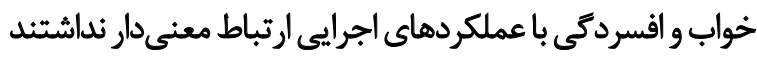

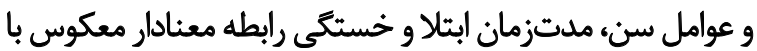

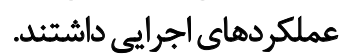

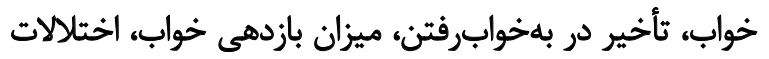

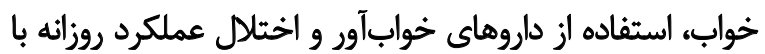

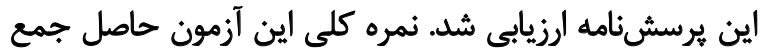

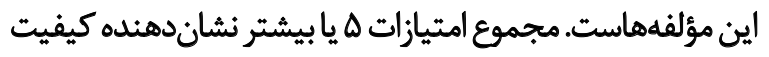

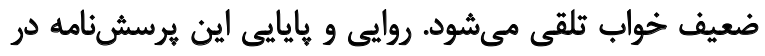

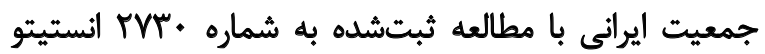

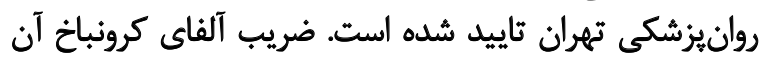

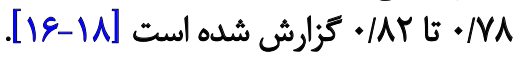

\section{مقياس ارزيابى خستكى ه ت}

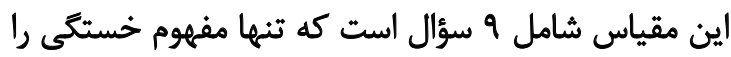

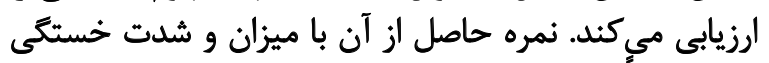

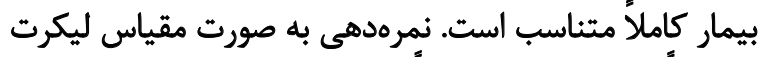

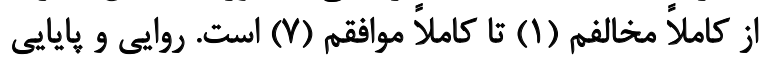

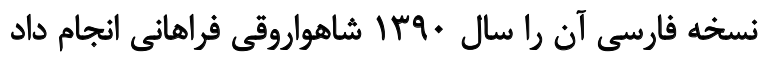

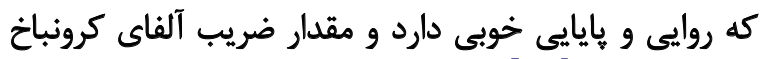

آن 199• است [19]

\section{آزمونهاى عملكرد اجرايى طبقهبئدى و توصيفى'}

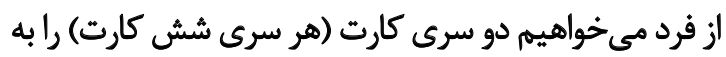

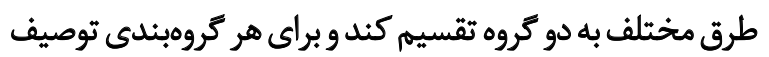

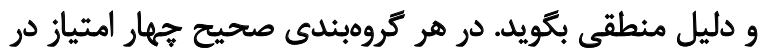

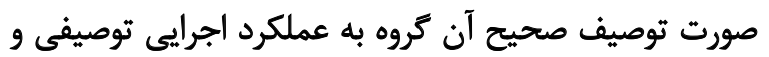

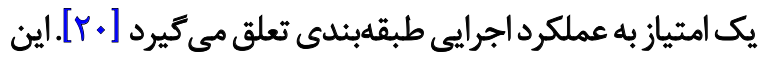

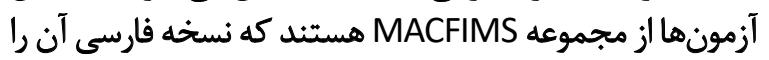

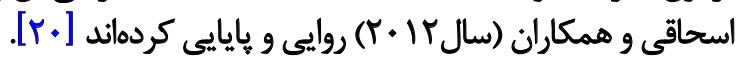

\section{معيار توصيفى وضعيت ناتوانى ^}

اين معيار، روش كمّى براى سنجش ناتوانى در بيمارى اماس

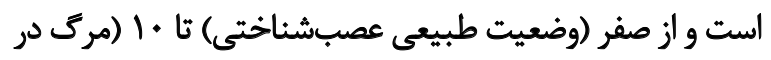

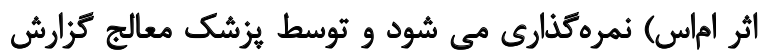

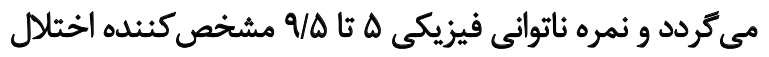

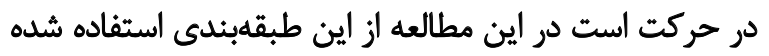

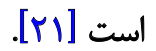

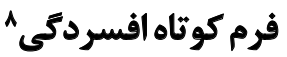

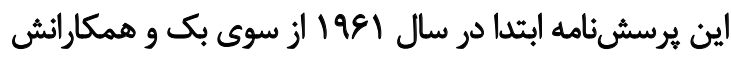

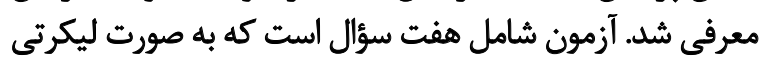

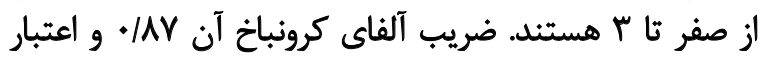

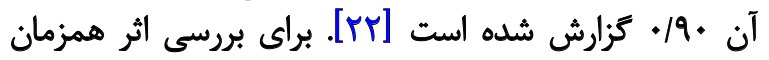

5. Fatigue Severity Scale

6. DKEF Sorting \& Descriptive

7. Expanded Disability Status Score (EDSS)

8. Beck Depression Inventory-Fast Screen 
جدول ا. توزيع فراوائى ويزّكى هاي فردى و بالينى آزمودنىها

\begin{tabular}{|c|c|c|c|}
\hline مرصد & تعداد & \multicolumn{2}{|c|}{ متغيرها } \\
\hline EV/E & PA & is & \multirow{3}{*}{ جنسيت } \\
\hline & & & \\
\hline$\pi / \%$ & ז & مرد & \\
\hline$r E / A$ & 19 & هجرد & \multirow{3}{*}{ وضعيث تأهل } \\
\hline$\Delta T / I$ & $r$ & مثأهل & \\
\hline$r M /$ & 10 & مطلقه و ييوه & \\
\hline $19 / V$ & if & سيكل و بايينتر & \multirow{3}{*}{ سطح تحصيلات } \\
\hline $\mathrm{rV} /$ & M & دييلم & \\
\hline$p q / r$ & ro & دانشكاهى & \\
\hline$r T / \Delta$ & 18 & عودكننده-فروكش & \multirow{3}{*}{ نوع بيمارى } \\
\hline rqg & M & ييشرونده اوليه & \\
\hline$P V / q$ & $m$ & يبيشرونده ثانويه & \\
\hline$\pi / \epsilon$ & M & بينايع & \multirow{4}{*}{ علامت شروع ييمارى } \\
\hline$\Delta T / I$ & rv & عضلانى & \\
\hline NQ & 8 & تعادل & \\
\hline $\mathrm{V} /$ & $\Delta$ & سركيجه وحالت تهوع & \\
\hline $18 / 9$ & ir & نداشتن اختملال خواب & \multirow{2}{*}{ اختالالات خواب } \\
\hline$\Lambda \% / 1$ & $\Delta q$ & اختلال خواب & \\
\hline$\Delta \& / N$ & r. & نداشتن افسردگى & \multirow{4}{*}{ افسردمى } \\
\hline $18 / 9$ & ir & خقيف & \\
\hline Tr/q & iv & هتوسط & \\
\hline$r / \Lambda$ & r & شليد & \\
\hline $\mathrm{N} / \mathrm{A} / \mathrm{q}$ & 81 & نمره بيشتر از P/ه & \multirow[b]{2}{*}{ ناتواثى فيزيكى } \\
\hline $\mid f / 1$ & 1. & نمره كمثر از F/ه & \\
\hline
\end{tabular}

توانبخننى

جدول r. ميانكين و انحراف معيار سن، مدتزمان ابتلاو خسئكى آزمودنىها

\begin{tabular}{|c|c|c|c|}
\hline حداكثر & حداقل & ميانكين +انحراف معيار & متغير \\
\hline$n$ & $r$ & $R / T M \pm V A P$ & سن \\
\hline$\pi$ & $1 / 0$ & $\mid r / A r \pm \Delta / A T$ & مدت ابثتلا (سال) \\
\hline er & 9 & $\Delta H / V \pm 1 \cdot / M$ & خستكي \\
\hline
\end{tabular}


جدول "ج نتيجه برازش مدل ركرسيون خطى تعميميافته بر عملكرد اجرايى (توصيفى و طبقهبندى)

\begin{tabular}{|c|c|c|c|c|c|c|}
\hline $\mathbf{P}$ & درجه آزادى & نمره والد & SE & مقدار ضريب & منيع تغييرات & متغير \\
\hline..$+ r$ & 1 & $q /+F^{\circ}$ & $.1 . \pi f$ & $-* / \cdot v \lambda$ & خستكى & عملكرد اجرايى \\
\hline $.1 . .1$ & 1 & $1 . / p V$ &.$/ \cdot r \Lambda$ & $-* / 1 \cdot r$ & سن سن س & توصيفى \\
\hline.$\% 1 r$ & $r$ & $1 . / 90$ & - & - & علامت شروع بيمارى & \\
\hline $.1 . .1$ & 1 & $1.1+1$ & $\% H$ & $-.1 . v a$ & خسنكى & طمالكرد اجرائي \\
\hline$<\cdot / . \cdot 1$ & 1 & $1 \% / .$. &.$/ .4 \lambda$ & $-+/ 1+r$ & سن & \\
\hline
\end{tabular}

جدولع. مقايسه زوجى ميانكين عملكرد اجرايى (طبقهبندى) در سطوح متغير اولين علامت شروع بيمارى

\begin{tabular}{|c|c|c|c|}
\hline $\mathbf{P}$ & SE & تفاوت ميانكينها & مقايسه تروههاى علامت شروع بيمارى \\
\hline $.1+.1$ & $F / 19$ & $\Delta / r \Delta$ & سركيجه و عضلانى \\
\hline 1.40 & $r / T r$ & $\mid r / * 1$ & سركيجه و بينايى \\
\hline $.1 . .1$ & $\Delta / \% \Delta$ & V/TY & سركيجه و تعادل \\
\hline $.1 . r A$ & $r / T Y$ & $g / M^{L}$ & عضلانى و يينايى \\
\hline .10 .1 & $r / 9 \Delta$ & $V / M$ & عضلانى و تعادل \\
\hline.$\% 1$ & $r / Q$ & $-t / N$ & ييناييى و تعادل \\
\hline
\end{tabular}

توانبخننى

كاهشدهنده سطح شناختى در نظر كرفته شده است [YS]]. در مطالعه حاضر بين جنسيت و عملكرد اجرايي رابطه معنادار

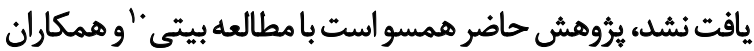

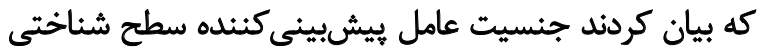

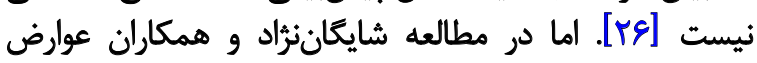

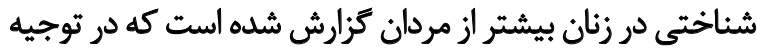

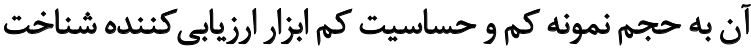

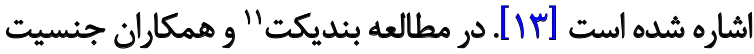

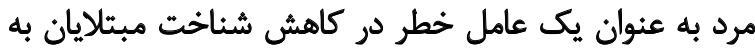

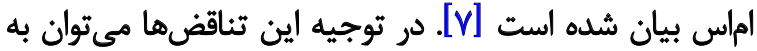

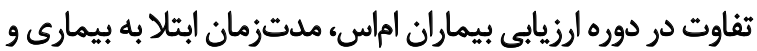

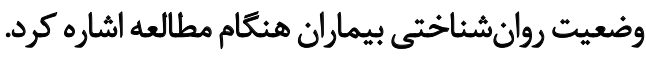

در مطالعه حاضر مدتزمان ابتلا همبستّى معكوس معنادار

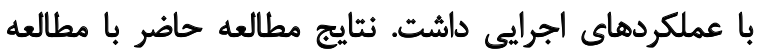

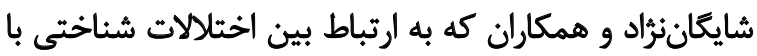

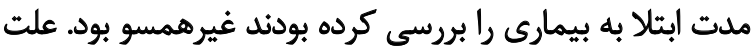

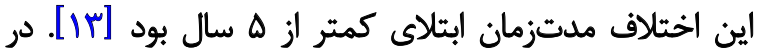

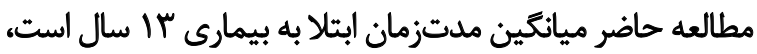
طولانى تربودن مدتزمان ابتلادر اين مطالعه نسبت بهانه مطالعات بات العات العات
عملكرد اجرايى (طبقهبندى) با اولين علامت شروع بيمارى

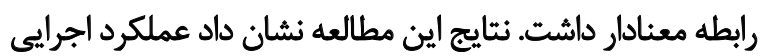

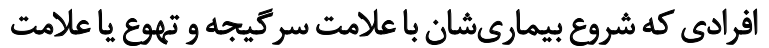

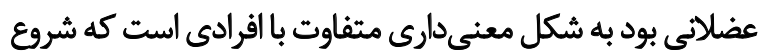

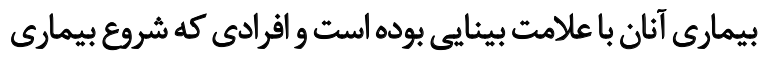

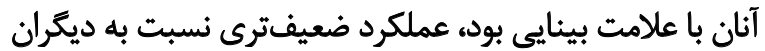

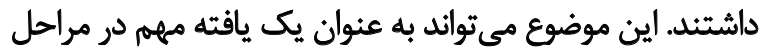

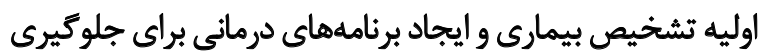
از عوارض شناختى مدنظر باشد.

طبق بررسىها در اين يُروهش، مطالعهاي در زمينه بررسى

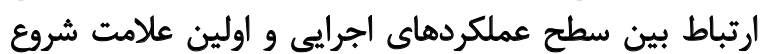

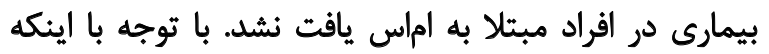

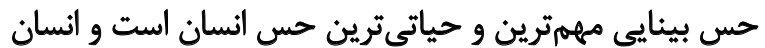

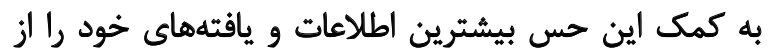

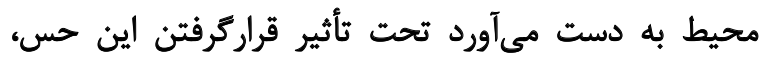

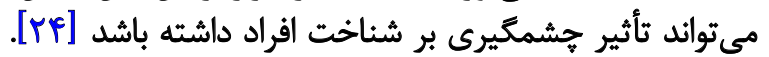

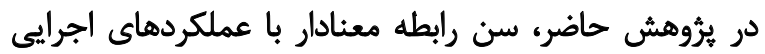

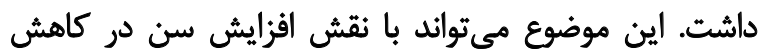

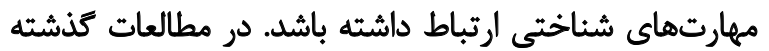

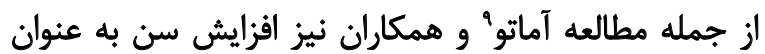


همجينين در مطالعه بداقى و همكاران كه عملكردهاى اجرايي

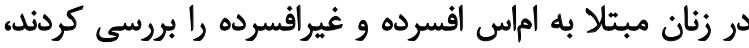

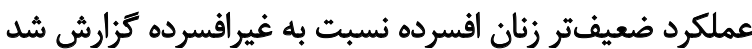

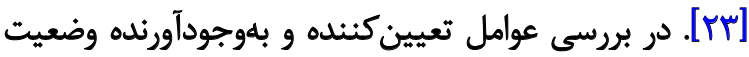

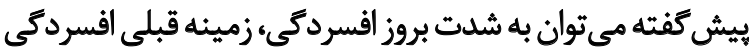

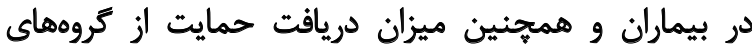
حمايت كثئده فرد افسرده در بيمارى اماس اشاره دئ دردات در اين يروهش بين ناتوانى فيزيكى و عملكردهاى اجرايى رابطه

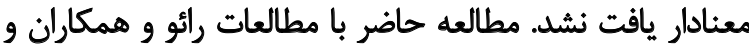

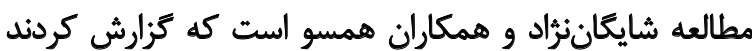

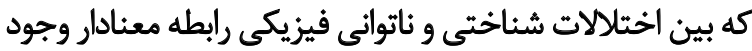

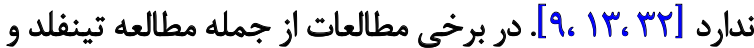

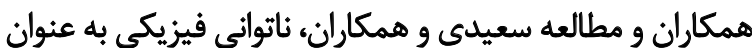

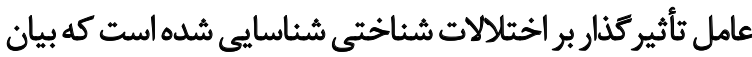

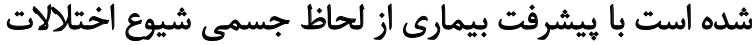

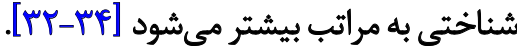

اين موضوع مى تواند به علت ماهيت متفاوت مقياسهاي

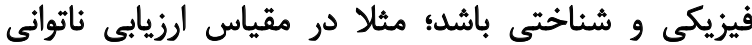

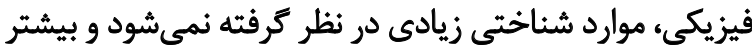

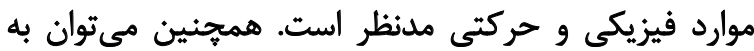

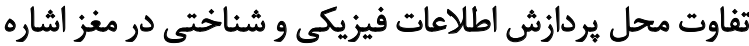

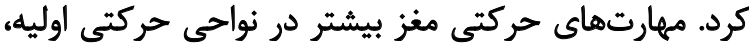

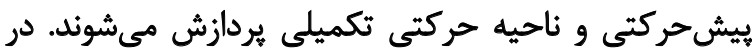

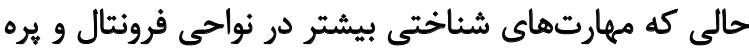

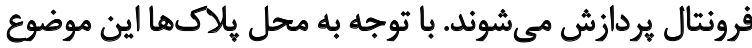

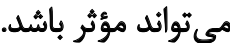

در اين يروهش بين وضعيت خواب افراد مبتلابه اماس و سطح

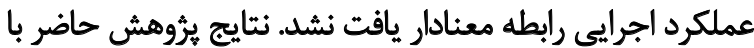

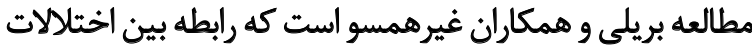

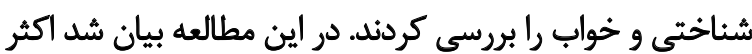

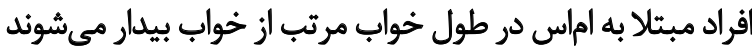

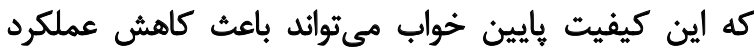

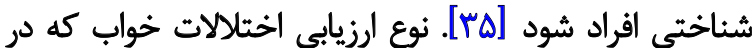

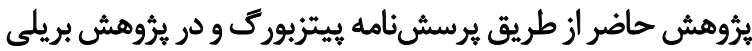

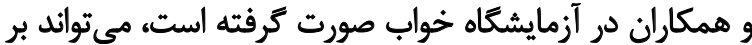

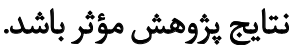

\section{نتيجليَّيرى}

با توجه به نتايج اين مطالعه مبنى بر وجود رابطه معنادار

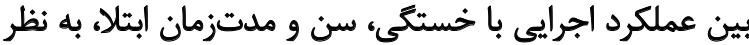

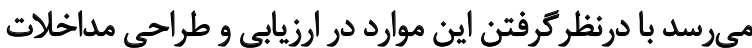

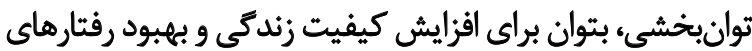
تطابقى كام مؤثرى برداشت بتران.
قبل مىتوائد باعث اين اختلاف شده باشد كه هرجه از زمان

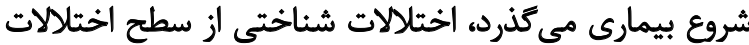

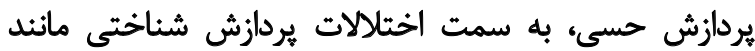

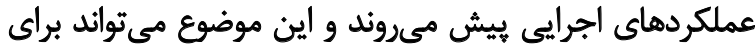

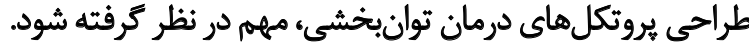
در بروهش حاضر، خسثتى رابطه معكوس با وضعيت عملكرد

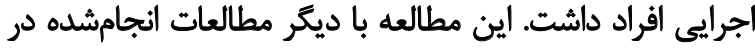

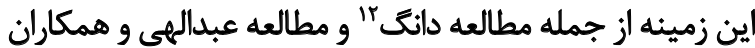

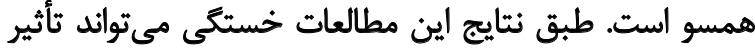

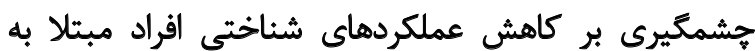

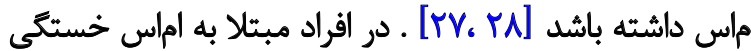

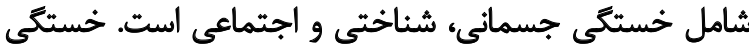

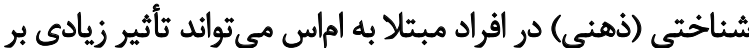

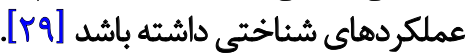
خستگى با تغيير فرايندهاي متابوليكى همراه است. فعاليت

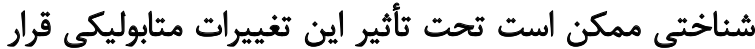

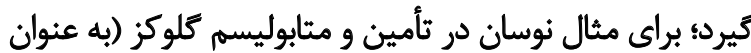

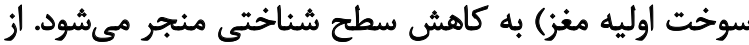

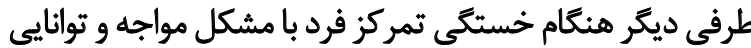

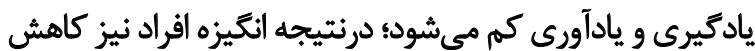

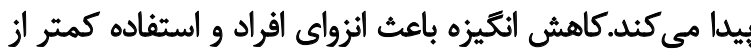

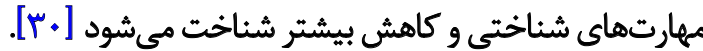

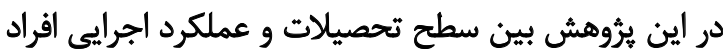

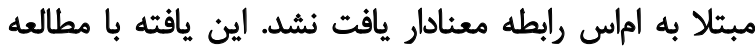

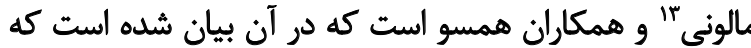

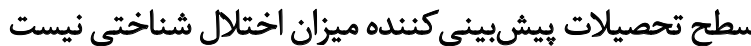

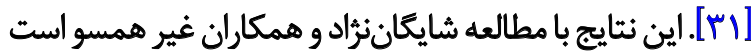

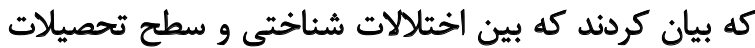

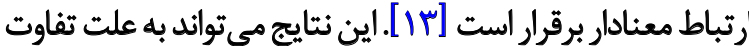
در نوع ابزارهاى ارزيابى شناختى باشد. در يروهش حاضر بين افسردگى و عملكردهاى اجرايى افراد

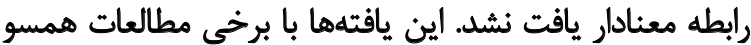

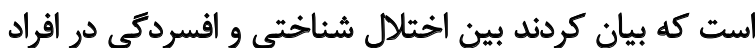

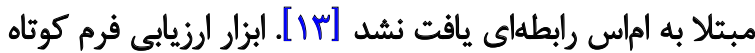

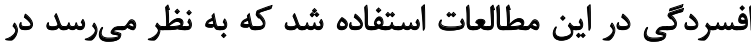

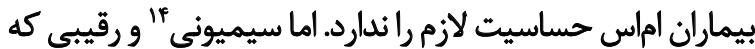

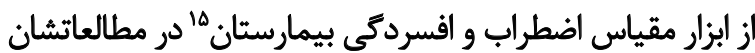

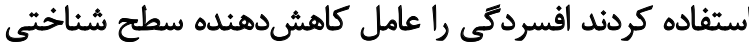

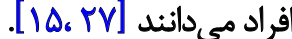

12. Duong

13. Maloni

14. Simioni

15. Hospital Anxiety and Depression scale (HAD) 
بيشنهاد مي شود عملكرد اجراييى افراد مبتلا به اماس با افراد

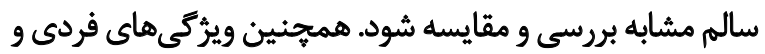

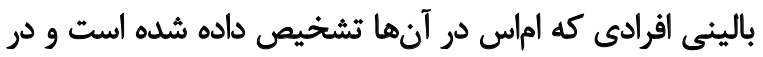
عملكرد اجرايى اختلال ندارند، بررسى شود آنها تشخي

از محدوديتهاى اين يُروهش، طولاتىيودن روند ارزيابي و

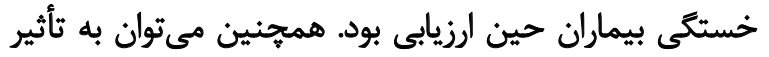

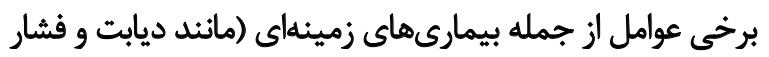

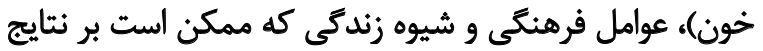
آزمونهاى شناختى مؤثر باشد، اشاره كرد.

مالاحظات اخلاقي

يبيروى ازٔ اصول اخلاق يثوهش

اين مطالعه را كميته اخلاق دانشَاه علوم بهزيستى و توانبخشى با كد IR.USWR.REC.1396.106 تأييد كرده است.

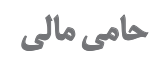

ثرثوهش حاضر بركرفتهاز يايان نامه كارشناسى ارشدمريم يوراميرى

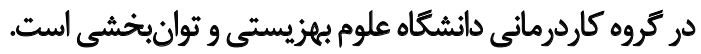

$$
\text { مشاركت ثويسند متكان }
$$

ايدهسازى: نازيلا اكبرفهيمى، مجتبى عظيميان؛ مفهومسازى:

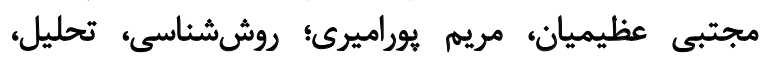

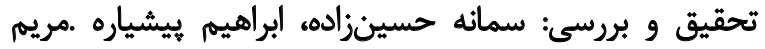

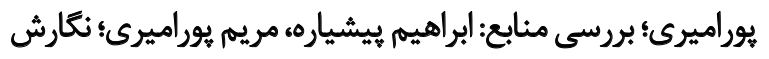

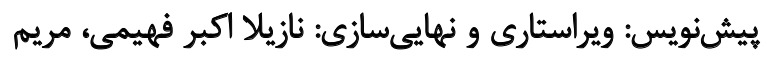

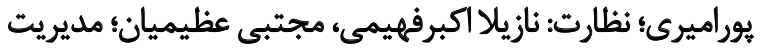
يروزه: نازيلاكبرفهيمى، مجتبى عظيميان.

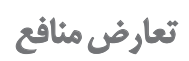

بنابر اظهار نويسندكانء اين مقاله تعارض منافع ندارد.

$$
\text { تشكر و قدردانى }
$$

نويسندكان بر خود لازم ميدانيد از يرسنل واحد كاردرماني

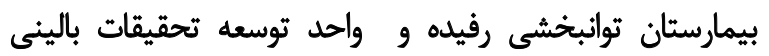
بيمارستان توانبخشى رفيده مراتب تشكر وقدردانى خود را ابراز كنيند. 


\section{References}

[1] Chiaravalloti ND, DeLuca J. Cognitive impairment in multiple sclerosis. The Lancet Neurology. 2008; 7(12):1139-51. [DOI:10.1016/S1474-4422(08)70259-X] [PMID]

[2] Abedini M, Paksersht M, Rafiei A, Valadan R, Amjadi O, Khajavi $R$, et al. [Demographic and clinical characteristics of multiple sclerosis (Persian)]. Journal of Mazandaran University of Medical Sciences. 2016; 25(132):13-22.

[3] Shiri V, Emami M, Shiri E. [Investigating the relationship between selective attention and cognitive flexibility with balance in patients with relapsing-remitting multiple sclerosis (Persian)]. Archives of Rehabilitation. 2018; 18(4):296-305. [DOI:10.21859/jrehab.18.4.4]

[4] Pakniya N, Bahmani B, Dadkhah A, Azimian M, Naghiyaee M, Masudi SR. Effectiveness of cognitive existential approach on decreasing demoralization in women with multiple sclerosis. Iranian Rehabilitation Journal. 2015; 13(4):28-33.

[5] Kidd D, Barkhof F, McConnell R, Algra P, Allen I, Revesz T. Cortical lesions in multiple sclerosis. Brain. 1999; 122(1):17-26. [DOI:10.1093/brain/122.1.17]

[6] Ge Y, Grossman RI, Udupa JK, Babb JS, Kolson DL, McGowan $J C$. Magnetization transfer ratio histogram analysis of gray matter in relapsing-remitting multiple sclerosis. American Journal of Neuroradiology. 2001; 22(3):470-5. [PMID]

[7] Benedict RH, Zivadinov R. Risk factors for and management of cognitive dysfunction in multiple sclerosis. Nature Reviews Neurology. 2011; 7(6):332-42. [DOI:10.1038/nrneurol.2011.61]

[8] Bagert B, Camplair P, Bourdette D. Cognitive dysfunction in multiple sclerosis. CNS Drugs. 2002; 16(7):445-55. [DOI:10.2165/00023210-200216070-00002]

[9] Rao SM, Leo GJ, Bernardin L, Unverzagt F. Cognitive dysfunction in multiple sclerosis. I. Frequency, patterns, and prediction. Neurology. 1991; 41(5):685-91. [DOI:10.1212/ WNL.41.5.685]

[10] Rao SM. Neurobehavioral aspects of multiple sclerosis. Oxford: Oxford University Press; 1990

[11] Benedict RH, Fischer JS, Archibald CJ, Arnett PA, Beatty WW, Bobholz J, et al. Minimal neuropsychological assessment of MS patients: A consensus approach. The Clinical Neuropsychologist. 2002; 16(3):381-97. [DOI:10.1076/clin.16.3.381.13859]

[12] Amato MP, Ponziani G, Pracucci G, Bracco L, Siracusa G, Amaducci $L$. Cognitive impairment in early-onset multiple sclerosis: Pattern, predictors, and impact on everyday life in a 4-year follow-up. Archives of Neurology. 1995; 52(2):168-72. [DOI:10.1001/archneur.1995.00540260072019]

[13] Shaygannejad V, Afshar H. The frequency of cognitive dysfunction among multiple sclerosis patients with mild physical disability. Journal of Isfahan Medical School. 2012; 29(167):17.

[14] Evans DA, Beckett LA, Albert MS, Hebert LE, Scherr PA, Funkenstein $\mathrm{HH}$, et al. Level of education and change in cognitive function in a community population of older persons.
Annals of Epidemiology. 1993; 3(1):71-7. [DOI:10.1016/10472797(93)90012-S]

[15] Simioni S, Ruffieux C, Bruggimann L, Annoni J, Schluep M Cognition, mood and fatigue in patients in the early stage of multiple sclerosis. Swiss Medical Weekly. 2007; 137(3536):496-501.

[16] Khajavi D, Khan Mohammadi R. [The effect of "green exercise" on improving the sleep quality of female elderly without regular physical activity in Arak City (Persian)]. Studies of Women and Family. 2016; 3(2):7-32.

[17] Farrahi J, Nakhaee N, Sheibani V, Garrusi B, Amirkafi A. Psy chometric properties of the Persian version of the Pittsburgh Sleep Quality Index addendum for PTSD (PSQI-A). Sleep and Breathing. 2009; 13:259. [DOI:10.1007/s11325-008-0233-3]

[18] Farhadi Nasab A, Azimi H. [Study of patterns and subjective quality of sleep and their correlation with personality traits among medical students of Hamadan University of Medical Sciences (Persian)]. Avicenna Journal of Clinical Medicine. 2008; 15(1):11-5.

[19] Farahani ShA, Azimian Fallahpour M, Karimloo M. [Assess the reliability of the Persian version Fatigue Severity Scale in patients with multiple sclerosis (Persian)]. Rehabilitation. 2010; 10(4):46-51.

[20] Eshaghi A, Riyahi-Alam S, Roostaei T, Haeri G, Aghsaei A Aidi MR, et al. Validity and reliability of a Persian translation of the Minimal Assessment of Cognitive Function in Multiple Sclerosis (MACFIMS). The Clinical Neuropsychologist. 2012 26(6):975-84. [DOI:10.1080/13854046.2012.694912]

[21] Goodkin DE, Cookfair D, Wende K, Bourdette D, Pullicino $P$, Scherokman $B$, et al. Inter-and intrarater scoring agreement using grades 1.0 to 3.5 of the Kurtzke Expanded Disability Status Scale (EDSS). Neurology. 1992; 42(4):859-63. [DOI:10.1212/WNL.42.4.859]

[22] Scogin F, Beutler L, Corbishley A, Hamblin D. Reliability and validity of the short form Beck Depression Inventory with older adults. Journal of Clinical Psychology. 1988; 44(6):853-7. [DOI:10.1002/1097-4679(198811)44:63.0.CO;2-7]

[23] Bodaghi E, Moradi A, Jafar H, Alipour F, Shahsavand Baghdadi T. [Comparison of executive functions in women with MS comorbid with and without depression and normal women (Persian)]. Neuropsychology. 2016; 2(5):37-54

[24] Bagheri S, Alireza D, Maryam Qalaji. [Evaluation of intensity of lighting and its relation with visual fatigue of students in study rooms of Zabol University of Medical Sciences in 1396 (Persian)]. Occupational Hygiene and Health Promotion Journal. 2017; 1(2):154-63.

[25] Amato MP, Ponziani G, Siracusa G, Sorbi S. Cognitive dysfunction in early-onset multiple sclerosis: A reappraisal after 10 years. Archives of Neurology. 2001; 58(10):1602-6. [DOI:10.1001/archneur.58.10.1602]

[26] Beatty WW, Goodkin DE, Hertsgaard D, Monson N. Clinical and demographic predictors of cognitive performance in multiple sclerosis: Do diagnostic type, disease duration, and disability matter? Archives of Neurology. 1990; 47(3):305-8. [DOI:10.1001/archneur.1990.00530030081019] 
[27] Raghibi M, Khosravi M. [Comparing the cognitive function in multiple sclerosis patients (Persian)]. Journal of Research in Medical Sciences. 2012; 14(2):45-8.

[28] Soleimani S, Tajoddini E. [Effectiveness of cognitive behavioral therapy based on fatigue on dimensions of mental fatigue in patients with multiple sclerosis: A case study (Persian)]. 2016; 8(2):13-21.

[29] Azimian M, Akbari Kamrani A. A glance on diagnosis and treatment of profound fatigue in multiple sclerosis. Iranian Rehabilitation Journal. 2007; 5(1):41-6.

[30] Krupp LB, Elkins LE. Fatigue and declines in cognitive functioning in multiple sclerosis. Neurology. 2000; 55(7):934-9. [DOI:10.1212/WNL.55.7.934]

[31] Maloni H. Cognitive impairment in multiple sclerosis. The Journal for Nurse Practitioners. 2018; 14(3):172-7. [DOI:10.1016/j.nurpra.2017.11.018]

[32] Saeedi MT, Ghoreyshi Rad F. [Clock crawing test: Screening of cognitive dysfunction in patients with multiple sclerosis (Persian)]. Journal of Isfahan Medical School. 2014; 31(268):221627.

[33] Tinnefeld M, Treitz FH, Haase CG, Wilhelm H, Daum I, Faustmann PM. Attention and memory dysfunctions in mild multiple sclerosis. European archives of Psychiatry and Clinical Neuroscience. 2005; 255(5):319-26. [DOI:10.1007/s00406005-0565-y]

[34] Rao SM, Leo GJ, Ellington L, Nauertz T, Bernardin L, Unverzagt F. Cognitive dysfunction in multiple sclerosis. II. Impact on employment and social functioning. Neurology. 1991; 41(5):6926. [DOI:10.1212/WNL.41.5.692]

[35] Braley TJ, Kratz AL, Kaplish N, Chervin RD. Sleep and cognitive function in multiple sclerosis. 2016; 39(8):1525-33. [DOI:10.5665/sleep.6012] [PMID] [PMCID] 